\title{
Diversity Exploiting MIMO-OFDMA Ranging
}

\author{
Jianqiang Zeng and Hlaing Minn (Contact Author) \\ Department of Electrical Engineering, University of Texas at Dallas \\ Email: $\{$ jxz051000, hlaing.minn $\} @$ utdallas.edu
}

\begin{abstract}
This paper addresses initial ranging (uplink synchronization and power control) for TDD MIMO OFDMA systems. Exploiting the channel knowledge from the downlink control channel together with initial power control, we develop a novel initial ranging method which provides multiuser and multi-antenna diversity gains and significant power saving for the subscriber stations. By means of ranging signal design, we develop efficient low-complexity algorithms for OFDMA ranging. The advantages of the proposed approach over existing methods in terms of ranging performance, energy saving, and complexity saving are illustrated by analytical and simulation results.
\end{abstract}

\section{INTRODUCTION}

In OFDMA systems [1] [2], uplink synchronization is accomplished by initial ranging process. The existing ranging methods [3]-[6] have a common disadvantage of high complexity. Furthermore, they addressed only for SISO OFDMA systems. In this paper we present a novel initial ranging method for time-division duplexing (TDD) MIMO OFDMA systems which provides multiuser and multi-antenna diversity gains while requiring only low-complexity algorithms for multiuser ranging signal detection, timing offset estimation and power estimation.

\section{System Descriptions And Signal Model}

Consider an uplink $M_{T} \times M_{R}$ MIMO OFDMA system with $N_{r}$ RSSs, $N_{d}$ data subscriber stations (DSSs) and $N$ subcarriers. After excluding DC and null subcarriers, the remaining subcarriers are grouped into $Q_{r}$ ranging subchannels and $Q_{d}$ data subchannels. The numbers of left and right null subcarriers are denoted by $\gamma_{L N}$ and $\gamma_{R N}$, respectively. Each ranging subchannel has $\gamma_{r}$ subcarriers and each data subchannel has $\gamma_{d}$ subcarriers. The indices of subcarriers corresponding to the $i$ th RSS and the $j$ th DSS are denoted as $\mathcal{J}_{i, r}$ and $\mathcal{J}_{j, d}$, respectively. The antenna index of SS and BS are denoted as $m_{t}$ and $m_{r}$, respectively.

In the subcarrier domain at the $m$ th OFDM symbol interval, the ranging code vector for the $i$ th RSS and the data vector for the $j$ th DSS are given by ${ }^{1}$

$$
\mathrm{X}_{i, \star}^{\left(m_{t}\right)}(n)= \begin{cases}A_{i, \star}^{\left(m_{t}\right)} C_{i, \star}^{\left(m_{t}\right)}(l), & n \in \mathcal{J}_{i, \star}(l) \\ 0, & l=0, \ldots, \gamma_{\star}-1 \\ 0, & \text { otherwise }\end{cases}
$$

where $C_{i, r}^{\left(m_{t}\right)}(l)$ and $C_{j, d}^{\left(m_{t}\right)}(l)$ are ranging and data symbols, respectively, with $\left|C_{i, r}^{\left(m_{t}\right)}(l)\right|=E\left[\left|C_{j, d}^{\left(m_{t}\right)}(l)\right|^{2}\right]=1$, $\left\{A_{i, r}^{\left(m_{t}\right)}, A_{j, d}^{\left(m_{t}\right)}>0\right\}$ are amplitude scaling factors, and the symbol index $m$ is omitted for simplicity. Denote the $N$ point inverse discrete Fourier transform $\left(\mathrm{IDFT}_{N}\right)$ of $\mathbf{X}_{i, \star}^{(m)}$ by

\footnotetext{
${ }^{1}$ In the rest of the paper, the subscript $\star$ denotes whether $r$ or $d$.
}

$\left[\mathrm{x}_{i, \star}^{(m)}(0), \ldots, \mathrm{x}_{i, \star}^{(m)}(N-1)\right]^{T}$. Then the time domain signal samples of the $i$ th RSS and the $j$ th DSS are given by

$$
\mathrm{x}_{i, \star}^{\left(m_{t}\right)}(n)= \begin{cases}\mathrm{x}_{i, \star}^{(m)}\left(l-N_{g}\right) & n=m\left(N+N_{g}\right)+l, \\ & l=0, \ldots, N+N_{g}-1 \\ & m=0, \ldots, M-1 \\ 0, & \text { otherwise }\end{cases}
$$

where $N_{g}$ is the number of the cyclic prefix (CP) samples and $M$ is the number of OFDM symbols per uplink frame.

We consider independent multi-path Rayleigh fading channels (quasi-static over the ranging period). The sample-spaced channel impulse response taps (including the transmit and receive filters) for the $i$ th $\mathrm{RSS}$ and the $j$ th DSS are denoted by $\left\{h_{i, r}^{\left(m_{t}, m_{r}\right)}(l)\right\}$ and $\left\{h_{j, d}^{\left(m_{t}, m_{r}\right)}(l)\right\}, l=0, \ldots, L-1$. The channel output samples of $m_{r}$ th antenna for the $i$ th RSS $\left\{y_{i, r}(n)\right\}$ and the $j$ th DSS $\left\{y_{j, d}(n)\right\}$ are given by

$$
y_{i, \star}^{\left(m_{r}\right)}(n)=\sum_{m_{t}=1}^{M_{T}} \sum_{l=0}^{L-1} h_{i, \star}^{\left(m_{t}, m_{r}\right)}(l) x_{i, \star}^{\left(m_{t}\right)}\left(n-l-d_{i, \star}\right),
$$

where $d_{i, r}$ and $d_{j, d}$ are the transmission delays for the $i$ th RSS and the $j$ th DSS, respectively. The maximum possible delay $d_{\max }$ is the round-trip transmission delay for a user at the cell boundary, and $N_{g} \geq d_{\max }+L-1$.

Then the time domain received signal of $m_{r}$ th antenna at the BS can be expressed as

$$
y^{\left(m_{r}\right)}(n)=\sum_{i=0}^{N_{r}-1} y_{i, r}^{\left(m_{r}\right)}(n)+\sum_{j=0}^{N_{d}-1} y_{j, d}^{\left(m_{r}\right)}(n)+\omega^{\left(m_{r}\right)}(n)
$$

where $\left\{\omega^{\left(m_{r}\right)}(n)\right\}$ are independent and identically-distributed (i.i.d.), circularly-symmetric complex Gaussian noise samples with zero mean and variance $\sigma_{\omega}^{2}$.

\section{Proposed Ranging Signal Design}

Consider an OFDMA system with $Q_{r}$ ranging subchannels and $\gamma_{r}$ contiguous subcarriers on each subchannels. The ranging subchannels are spread out over the whole frequency band with approximately equal spacing. The subcarrier assignment for the $i$ th subchannel $\left(i=0, \ldots, Q_{r}-1\right)$ is defined as

$$
\mathcal{J}_{i, r}=\left\{\gamma_{L N}+i \cdot \Delta+n: n=0, \ldots, \gamma_{r}-1\right\}
$$

where $\Delta=\left\lfloor\frac{N_{\text {used }}-\gamma_{r}}{Q_{r}-1}\right\rfloor$ and $N_{\text {used }}=\gamma_{r} Q_{r}+\gamma_{d} Q_{d}$. Note that $\left\{\mathcal{J}_{i, r}\right\}$ and $\left\{\mathcal{J}_{k, r}\right\}$ are disjoint for $i \neq k$.

The proposed ranging signal design has $N_{c}=\gamma_{r} / 2$ frequency domain orthogonal codes (e.g., Walsh codes) of length $N_{c}$ each. Each code is repeated as $C_{i, r}\left(0, \ldots, \frac{\gamma_{r}}{2}-1\right)=$ $C_{i, r}\left(\frac{\gamma_{r}}{2}, \ldots, \gamma_{r}-1\right)$ to form a length- $\gamma_{r}$ ranging code. Each RSS transmits a ranging code randomly chosen from the above $N_{c}$ orthogonal codes on the chosen ranging subchannel defined by $\mathcal{J}_{i, r}$ only at one time slot randomly chosen from the $M$ 
ranging time slots. In our proposed scheme, each RSS uses only one antenna for transmission and each ranging time slot is equal to one OFDM symbol interval. The total number of ranging opportunities is $N_{\text {total }}=Q_{r} \cdot N_{c} \cdot M$.

Note that the channel responses within a ranging subchannel of the proposed scheme (e.g., $\gamma_{r}=8$ adjacent subcarriers in an OFDMA system with $N=2048$ ) are almost the same. Hence, the proposed ranging signals still maintain orthogonality at the BS (under the condition of negligible frequency offsets), while the ranging signals from 802.16a/e suffer from loss of orthogonality due to the channel frequency selectivity.

\section{The Proposed Ranging Method}

Our proposed method first performs multi-user ranging signal detection, and then estimates timing offset for each detected RSS by utilizing the ranging signal detection results. Next, by combining the ranging signal detection results and the timing offsets estimation results, the received power of each detected RSS is estimated. The proposed ranging process is summarized in the following:

1) Each RSS estimates the channel power gains across the band by utilizing the pilots embedded in the downlink control frame received at each antenna. The RSS picks the antenna with the best ranging subchannel, i.e. with the largest $\sum_{m_{r}=1}^{M_{R}}\left|H_{i, r}^{\left(m_{t}, m_{r}\right)}\right|^{2}$, and adjusts the transmission power so that the received ranging signal power approximately equals to the target received ranging signal power $P_{r}=\left|A_{i}\right|^{2} \sum_{m_{r}=1}^{M_{R}}\left|H_{i, r}^{\left(m_{t}, m_{r}\right)}\right|^{2}$. If the adjusted transmission power is greater than the maximum allowable transmission power $P_{\max }$, then the RSS will not initiate the ranging process.

2) Each RSS randomly chooses one of the $N_{c}$ orthogonal codes and one of the $M$ ranging time slots, and then uses the selected antenna to transmit the ranging signal over the selected ranging subchannel in the selected ranging time slot.

3) At the BS, the receiver identifies whether or not a ranging code is transmitted, and extracts the timing and power information based on the detection results. The BS compares the estimated timing offset and power with the ranging requirements for each detected RSS. If they satisfy the requirements, BS informs RSS that ranging process is successful. If not, BS sends timing and power adjustment parameters and a "continue" status notification to RSS.

4) The RSSs with "success" status complete the ranging process, each of the RSSs with "continue" status will adjust the timing and power accordingly and randomly chooses a ranging code to continue the ranging process in the same ranging subchannel and time slot as the last ranging trial. The RSSs which do not find their ranging information in the ranging response message (i.e., the RSSs with collision) will each randomly choose a ranging code and a ranging time slot but use the same ranging subchannel to re-initiate the ranging process in the next frame or according to the truncated exponential backoff scheme.

When an RSS cannot detect the downlink control channel, or the ranging transmit power needed is larger than $P_{\max }$, the situation can be reflected in the received signal strength indicator at the RSS. This will alert the user to move around to get good channel to be able to start its ranging process.

\section{A. Multi-User Ranging Signal Detection}

At the BS, the frequency domain signal of the $m_{r}$ th antenna can be expressed as

$$
\begin{aligned}
Y^{\left(m_{r}\right)}(k)= & \sum_{i=1}^{N_{r}} X_{i, r}(k) H_{i, r}^{\left(m_{t}, m_{r}\right)}(k)+\sum_{j=1}^{N_{d}} X_{j, d}(k) H_{j, d}^{\left(m_{t}, m_{r}\right)}(k) \\
& +W^{\left(m_{r}\right)}(k) \\
\text { where } \quad & H_{i, \star}^{\left(m_{t}, m_{r}\right)}(k)=e^{\frac{j 2 \pi k d_{i, \star}}{N}} \widetilde{H}_{i, \star}^{\left(m_{t}, m_{r}\right)}(k) \\
W^{\left(m_{r}\right)}(k)= & \frac{1}{\sqrt{N}} \sum_{l=0}^{N-1} \omega^{\left(m_{r}\right)}\left(m\left(N+N_{g}\right)+N_{g}+l\right) e^{\frac{-j 2 \pi l k}{N}}
\end{aligned}
$$

Since SS transmits from only one antenna, we drop the SS antenna index $m_{t}$ for simplicity. For $m_{r}$ th receive antenna, the BS correlates the received frequency domain symbols of every $i$ th ranging subchannel with each $j$ th possible ranging code at each ranging time slot as

$$
\begin{aligned}
D 1_{i, j}^{\left(m_{r}\right)} & =\sum_{l=0}^{\frac{\gamma_{r}}{2}-1} Y^{\left(m_{r}\right)}\left(\mathcal{J}_{i, r}(l)\right) C_{j, r}^{*}(l) \\
D 2_{i, j}^{\left(m_{r}\right)} & =\sum_{l=\frac{\gamma_{r}}{2}}^{\gamma_{r}-1} Y^{\left(m_{r}\right)}\left(\mathcal{J}_{i, r}(l)\right) C_{j, r}^{*}\left(l-\frac{\gamma_{r}}{2}\right) .
\end{aligned}
$$

Then the decision variable $D_{i}$ for the RSS detection at the $i$ th ranging subchannel is defined as

$$
D_{i}=\sum_{m_{r}=1}^{M_{R}}\left(\left|D 1_{i}^{\left(m_{r}\right)}\right|^{2}+\left|D 2_{i}^{\left(m_{r}\right)}\right|^{2}\right)
$$

where the ranging code index $j$ is omitted for simplicity.

In developing our RSS detector, we consider only 0 and 1 RSS for each ranging opportunity, since the probability of 2 or more RSSs choosing the same ranging opportunity is very small. Each RSS adjusts its transmission power to compensate for the power loss due to channel and hence $\left|A_{i}\right|^{2} \sum_{m_{r}=1}^{M_{R}}\left|H_{i, r}^{\left(m_{r}\right)}\left(\mathcal{J}_{i, r}(l)\right)\right|^{2} \cong P_{r}$ for all $i$. The probability density function (PDF) of the $D_{i}$ for 0 and 1 RSS cases are central and non-central Chi-square with $n=4 M_{R}$ degrees of freedom, given by

$$
f_{D_{i}}(x)= \begin{cases}\frac{1}{2^{\frac{n}{2}} \sigma^{n} \Gamma\left(\frac{n}{2}\right)} x^{\frac{n}{2}-1} e^{-\frac{x}{2 \sigma^{2}}}, & 0 \mathrm{RSS} \\ \frac{1}{2 \sigma^{2}}\left(\frac{x}{s_{1}^{2}}\right)^{\frac{n-2}{4}} e^{-\frac{s_{1}^{2}+x}{2 \sigma^{2}}} I_{\frac{n}{2}-1}\left(\frac{\sqrt{x} s_{1}}{\sigma^{2}}\right), & 1 \mathrm{RSS}\end{cases}
$$

where $\sigma^{2}=\frac{\gamma_{r}}{4} \sigma_{w}^{2}$ and $s_{1}^{2}$ is shown in (13).

Then the presence or absence of the ranging code on a ranging opportunity (denoted by $n_{\mathrm{RSSs}}=1$ or 0 , respectively) is determined by

$$
n_{\mathrm{RSS}}=\underset{n=0,1}{\operatorname{argmax}}\left\{P_{n} \cdot f_{D_{i}}\left(D_{i} \mid n \text { RSSs }\right)\right\}
$$

where $\left\{P_{n}\right\}$ representing the probability of $n$ RSSs in the same ranging opportunity can be replaced with pre-defined design values. This detection is equivalent to

$$
n_{\mathrm{RSS}}= \begin{cases}0, & \text { if } D_{i}<\eta \\ 1, & \text { else }\end{cases}
$$

where $\eta$ is the crossover point of $P_{0} \cdot f_{D_{i}}\left(D_{i} \mid 0 \mathrm{RSS}\right)$ and $P_{1} \cdot f_{D_{i}}\left(D_{i} \mid 1\right.$ RSS $)$. 


\section{B. Timing Offset Estimation}

For the collision-free $i$ th RSS, $D 1_{i}$ and $D 2_{i}$ are given by

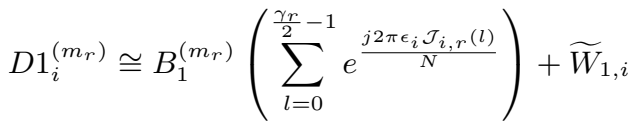

$$
\begin{aligned}
& D 2_{i}^{\left(m_{r}\right)} \cong B_{2}^{\left(m_{r}\right)} e^{\frac{j 2 \pi \epsilon_{i} \frac{\gamma_{r}}{2}}{N}}\left(\sum_{l=0}^{\frac{\gamma_{r}}{2}-1} e^{\frac{j 2 \pi \epsilon_{i} \mathcal{J}_{i, r}(l)}{N}}\right)+\widetilde{W}_{2, i}
\end{aligned}
$$

where $B_{1}^{\left(m_{r}\right)} \cong B_{2}^{\left(m_{r}\right)}=A_{i} \widetilde{H}_{i, r}^{\left(m_{r}\right)}\left(\mathcal{J}_{i, r}(l)\right), \epsilon_{i}$ is the timing offset of the $i$ th RSS, and $\left\{\widetilde{W}_{1, i}, \widetilde{W}_{2_{i}}\right\}$ are i.i.d. zeromean complex Gaussian random variables with a variance of $\sigma_{w}^{2} \gamma_{r} / 2$. Since $B_{1}^{\left(m_{r}\right)} \cong B_{2}^{\left(m_{r}\right)}$, the timing offset can be estimated as

$$
\hat{\epsilon}_{i}=\operatorname{round}\left\{\frac{N \cdot \text { angle }\left\{\sum_{m_{r}=1}^{M_{R}} D 2_{i}^{\left(m_{r}\right)}\left(D 1_{i}^{\left(m_{r}\right)}\right)^{*}\right\}}{\pi \gamma_{r}}\right\} .
$$

Since $0 \leq \epsilon_{i} \leq d_{\max }$, for occasional cases of $\hat{\epsilon}_{i}>d_{\max }$, we can set $\hat{\epsilon}_{i}=d_{\max }$. The above estimator provides a significant complexity reduction over the existing methods [3] [4] [5].

\section{RSS Power Estimation}

In one ranging slot, if only one code is detected, define

$$
\begin{array}{r}
P 1_{i}^{\left(m_{r}\right)}=\frac{2}{\gamma_{r}} \sum_{l=0}^{\frac{\gamma_{r}}{2}-1} Y^{\left(m_{r}\right)}\left(\mathcal{J}_{i, r}(l)\right) C_{j, r}^{*}(l) e^{\frac{-j 2 \pi \hat{\epsilon}_{i} \mathcal{J}_{i, r}(l)}{N}} \\
P 2_{i}^{\left(m_{r}\right)}=\frac{2}{\gamma_{r}} \sum_{l=\frac{\gamma_{r}}{2}}^{\gamma_{r}-1} Y^{\left(m_{r}\right)}\left(\mathcal{J}_{i, r}(l)\right) C_{j, r}^{*}\left(l-\frac{\gamma_{r}}{2}\right) e^{\frac{-j 2 \pi \hat{\epsilon}_{i} \mathcal{J}_{i, r}(l)}{N}} .
\end{array}
$$

and more than one distinct codes are detected, define

$$
P 1_{i}^{\left(m_{r}\right)}=\frac{2}{\gamma_{r}} D 1_{i}^{\left(m_{r}\right)}, \quad P 2_{i}^{\left(m_{r}\right)}=\frac{2}{\gamma_{r}} D 2_{i}^{\left(m_{r}\right)} .
$$

Then the power estimator is given by

$$
\hat{P}=\sum_{m_{r}=1}^{M_{R}} \frac{\left(\left|P 1_{i}^{\left(m_{r}\right)}\right|^{2}-\frac{\sigma_{w}^{2}}{\frac{\gamma r}{2}}\right)+\left(\left|P 2_{i}^{\left(m_{r}\right)}\right|^{2}-\frac{\sigma_{w}^{2}}{\frac{\gamma r}{2}}\right)}{2}
$$

where the noise variance $\sigma_{w}^{2}$ is assumed to be known (can be measured or estimated easily).

From (11) and (22), we know that $\hat{P}=\frac{2}{\gamma_{r}^{2}} D_{i}-\frac{4}{\gamma_{r}} \sigma_{w}^{2}$. So the mean of the power estimation is

$$
E[\hat{P}]=\frac{2}{\gamma_{r}^{2}}\left(8 \sigma^{2}+s_{1}^{2}\right)-\frac{4}{\gamma_{r}} \sigma_{w}^{2}=P_{r}
$$

and the variance of power estimation $\sigma_{\hat{P}}^{2}$ can be calculated as

$$
\sigma_{\hat{P}}^{2}=\left(\frac{2}{\gamma_{r}^{2}}\right)^{2}\left(16 \sigma^{4}+4 \sigma^{2} s_{1}^{2}\right)=\frac{4 \sigma_{w}^{4}}{\gamma_{r}^{2}}+\frac{2 \sigma_{w}^{2} P_{r}}{\gamma_{r}} .
$$

\section{Average Ranging Transmission Power}

We consider a multipath Rayleigh fading channel with $Q_{r}$ sample-spaced i.i.d. taps (having a uniform power delay profile) for analytical tractability. The proposed equi-spaced ranging subchannels can be approximately modeled as $Q_{r}$ cyclically equi-spaced ranging subchannels. Let $H_{i, k}^{\left(m_{t}, m_{r}\right)}$ denote channel gain of the $i$ th RSS $\left(i=0,1, \ldots, N_{R}-1\right)$ on the $k$ th $(k=0,1, \ldots, N-1)$ subcarrier. $\left\{H_{i, k}^{\left(m_{t}, m_{r}\right)}\right\}$ are circularly-symmetric complex Gaussian random variables with zero mean and unit variance. Then the average channel power gain of the $n$-th ranging subchannel for the $m_{t}$ th antenna of the $i$ th RSS is given by

$$
\begin{aligned}
G_{i, n}^{\left(m_{t}\right)} & =\quad \frac{\sum_{m_{r}=1}^{M_{R}} \sum_{k=\gamma_{r} \cdot n}^{\gamma_{r} \cdot(n+1)-1}\left|H_{i, k}^{\left(m_{t}, m_{r}\right)}\right|^{2}}{\gamma_{r}} \\
& \cong \sum_{m_{r}=1}^{M_{R}}\left|H_{i, \gamma_{r} \cdot n}^{\left(m_{t}, m_{r}\right)}\right|^{2}, n=0,1, \ldots, Q_{r}-1 .
\end{aligned}
$$

From (25), we find that $\left\{G_{i, n}^{\left(m_{t}\right)}, n=0,1, \ldots, Q_{r}-1\right\}$ are approximately i.i.d. Chi-square random variables with $2 M_{R}$ degree of freedom. The cumulative distribution function (CDF) of $G_{i, n}$ is given by

$$
F_{G}(g)=\left[1-e^{-g} \sum_{l=0}^{M_{R}-1} \frac{1}{l !}(g)^{l}\right] u(g)
$$

where $u(g)$ is the unit step function. In the proposed ranging method, the RSS will pick the antenna with the best channels based on the channel power gain $G_{i, n}^{\left(m_{t}\right)}$ defined in (25). Define

$$
Z_{i}=\max \left\{G_{i, n}^{\left(m_{t}\right)}: n=0,1, \ldots, Q_{r}-1, m_{t}=1, \ldots, M_{T}\right\} .
$$

Then the CDF of $Z_{i}$ is

$$
F_{Z_{i}}(z)=\left[1-e^{-z} \sum_{l=0}^{M_{R}-1} \frac{1}{l !}(z)^{l}\right]^{M_{T} Q_{r}} .
$$

Each RSS will adjust its transmission power such that

$$
P_{t, i} Z_{i}=P_{r}
$$

where $P_{t, i}$ is the adjusted transmission power of the $i$ th RSS and $P_{r}$ is the target received ranging signal power of an RSS at the BS. Following mobile WiMAX systems [2], in the following we use $M_{T}=M_{R}=2$. Then from (28) and (29), we obtain the PDF of $P_{t, i}$ as

$$
f_{P_{t, i}}(p)=\frac{P_{r}^{2}}{p^{3}} 2 Q_{r}\left(1-e^{-\frac{P_{r}}{p}}\left(1+\frac{P_{r}}{p}\right)\right)^{2 Q_{r}-1} e^{-\frac{P_{r}}{p}} .
$$

Then the mean of the ranging transmission power is given by (32), where $\Gamma$ is the upper incomplete gamma function defined by $\Gamma(a, x)=\int_{x}^{\infty} e^{-t} t^{a-1} d t$.

$$
s_{1}^{2}=\sum_{m_{r}=1}^{M_{R}}\left(\left|\sum_{l=0}^{\frac{\gamma_{r}}{2}-1} A_{i} H_{i, r}^{\left(m_{r}\right)}\left(\mathcal{J}_{i, r}(l)\right)\right|^{2}+\left|\sum_{l=\frac{\gamma_{r}}{2}}^{\gamma_{r}-1} A_{i} H_{i, r}^{\left(m_{r}\right)}\left(\mathcal{J}_{i, r}(l)\right)\right|^{2}\right) \cong 2\left(\frac{\gamma_{r}}{2}\right)^{2}\left|A_{i}\right|^{2} \sum_{m_{r}=1}^{M_{R}}\left|H_{i, r}^{\left(m_{r}\right)}\left(\mathcal{J}_{i, r}(l)\right)\right|^{2} \cong 2\left(\frac{\gamma_{r}}{2}\right)^{2} P_{r}
$$


TABLE I

SIMULATION PARAMETERS

\begin{tabular}{|c|c|}
\hline System channel BW (MHz) & 3 \\
\hline Sampling frequency $(\mathrm{MHz})$ & 3.36 \\
\hline FFT size $N$ & 2048 \\
\hline CP length $N_{g}$ & 128 \\
\hline Cell radius & $4.5 \mathrm{~km}$ \\
\hline Maximum RSS timing offset $d_{\max , \mathrm{r}}$ & 104 samples \\
\hline Maximum DSS timing offset $d_{\max , \mathrm{d}}$ & 32 samples \\
\hline Sub-carrier spacing & $1.64 \mathrm{kHz}$ \\
\hline Number of DC subcarriers & 1 \\
\hline Number of guard subcarriers, left & 184 \\
\hline Number of guard subcarriers, right & 183 \\
\hline Number of used subcarriers & 1680 \\
\hline Number of OFDM symbols in one uplink frame & 6 \\
\hline \multirow[t]{2}{*}{ Modulation } & BPSK for RSS \\
\hline & QPSK for DSS \\
\hline Number of data subcarriers per subchannel & 48 \\
\hline Number of channel Taps $L$ & 16 \\
\hline Timing requirement & 32 Samples \\
\hline Power requirement & $9.4 \mathrm{~dB}$ \\
\hline Residual normalized frequency offsets & {$[-0.02,0.02]$} \\
\hline Simulation runs & 5000 \\
\hline
\end{tabular}
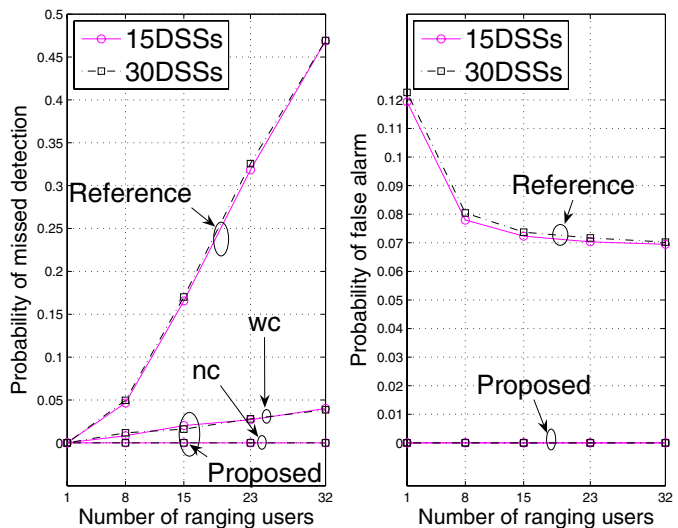

Fig. 1. The probabilities of missed detection and false alarm at the first ranging frame

\section{Simulation Results and Discussion}

\section{A. Simulation Parameters}

For comparison, the ranging code detector and timing estimator in [3] with the ranging signals from the IEEE 802.16e are used as the reference method. The system parameters, selected from [2], are summarized in Table I. The reference method is applied in SISO OFDMA, while the proposed method is applied in MIMO OFDMA. For the proposed method, the ranging channel is composed of 16 ranging subchannels with 8 contiguous subcarriers each. The number of orthogonal codes (and the code length) is $N_{c}=4$. The total number of ranging opportunities in the proposed method is $N_{\text {total }}=16 \cdot 4 \cdot 6=384$.

For the reference method, each RSS transmits one selected CDMA code twice on a single ranging channel that consists of 144 non-contiguous subcarriers over two consecutive symbols.
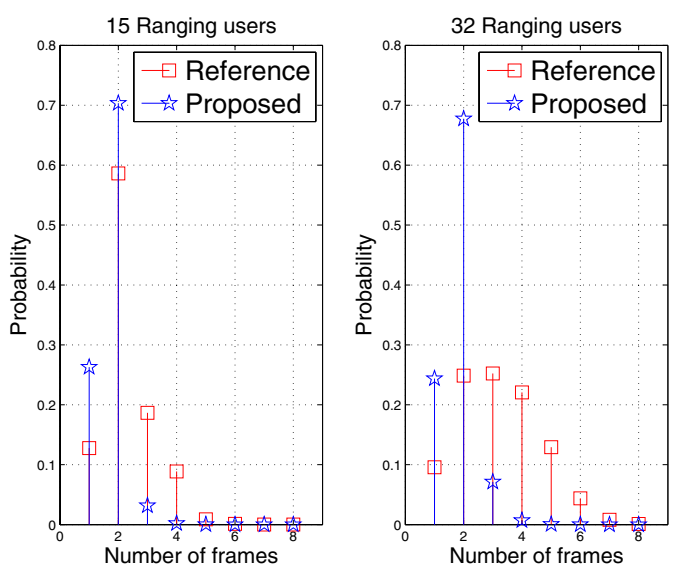

Fig. 2. The probability distribution of the number of frames required for a successful ranging

In order to reduce the simulation time for the reference method, we use 32 ranging codes which is equal to the considered maximum number of RSSs and we assume there is no collision in the reference method. Since there is no power estimator provided in [3], we assume perfect power estimation for the reference method. Hence the performance of the reference method presented will be slightly optimistic. For simplicity in evaluation, we assume that all $N_{r}$ RSSs attempt their ranging simultaneously at the first ranging frame, there are no new RSSs until their ranging processes are completed, and collided RSSs retry their ranging in the next frame.

For the reference method, the initial ranging transmission power on each ranging subcarrier is set $10 \mathrm{~dB}$ above the noise power $\left(\sigma_{w}^{2}\right)$ on a subcarrier. So the total ranging transmission power over 144 ranging subcarriers normalized by $\sigma_{w}^{2}$ is 10 $\log _{10}(1440) \mathrm{dB}$. In the proposed method, the target received total ranging signal power of an RSS is set the same as the statistical average of the total received ranging signal power of an RSS in the reference method, i.e., $P_{r} / 2 \sigma_{w}^{2}=1440 / 8$. Under this setting, the reference method utilizes twice the ranging transmission energy of the proposed method without power control and multiuser diversity since the former transmits on two OFDM symbols while the latter sends on one OFDM symbol interval.

\section{B. Simulation Results and Discussions}

Fig. 1 shows that probabilities of missed detection $P_{\text {miss }}$ and false alarm $P_{\text {false }}$ evaluated under collision-free condition as well as for the case with collision. $P_{\text {miss }}$ is defined as $E\left[\frac{D_{m}}{N_{r}}\right]$ where $D_{m}$ is the number of RSSs whose transmitted ranging signals are not detected at the BS and $N_{r}$ is the total number of RSSs. $P_{\text {false }}$ is defined as $E\left[\frac{D_{a}}{N_{\text {total }}-N_{r}}\right]$ where $D_{a}$ is the number of ranging signals which are detected at the BS but are not transmitted from any RSSs. For the proposed method

$$
E\left[P_{t, i}\right]=\int_{0}^{P_{\max }} p f_{P_{t, i}}(p) d p=2 P_{r} Q_{r} \sum_{k=0}^{2 Q_{r}-1}\left(\begin{array}{c}
2 Q_{r}-1 \\
k
\end{array}\right)(-1)^{k} \sum_{m=0}^{k}\left(\begin{array}{c}
k \\
m
\end{array}\right)\left\{(k+1)^{-(m+1)} \Gamma\left(m+1,(k+1) \frac{P_{r}}{P_{\max }}\right)\right\}
$$




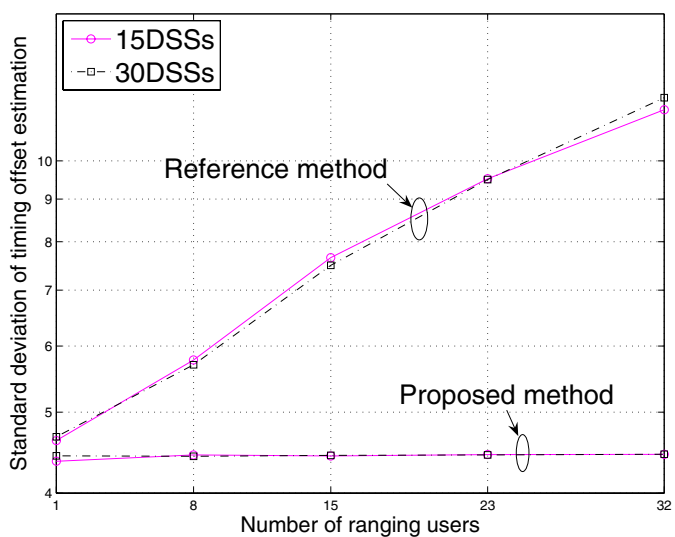

Fig. 3. Timing offset estimation performance comparison

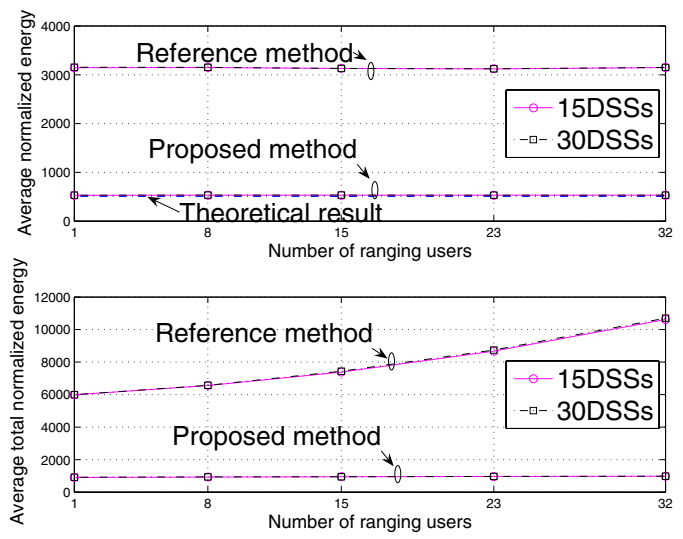

Fig. 4. Average normalized ranging transmission energy comparison

in the figure, "wc" and "nc" denote the cases with collision and without collision, respectively. The reference method is evaluated under collision free condition. The proposed method generally performs much better than the reference method. No false alarms are observed for the proposed method under both considered conditions. For the collision-free case (the same condition as the reference method), neither missed detection nor false alarm is observed for the proposed method. There are some missed detections observed in the "wc" case, this is because the $P_{\text {miss }}$ defined above counts all undetected RSSs with or without collision.

Fig. 2 shows the probability distribution of the number of frames required for an RSS to complete a successful ranging. For 15 RSSs case, the simulation results show that about $95 \%$ of the RSSs in the proposed method $(70 \%$ in the reference method) can finish the ranging process in two frames. For 32 RSSs case, we can see that the proposed method yields a greater advantage ( $90 \%$ versus $35 \%$ ).

Fig. 3 shows the standard deviation of the timing offset estimation. The true timing offsets for RSSs and DSSs are taken randomly from the interval $\left[0, d_{\max , \mathrm{r}}\right]$ and $\left[0, d_{\max , \mathrm{d}}\right]$, respectively. The results show that both methods are robust to the DSS interference. The proposed estimator is also robust to the RSS interference, while the performance of the reference estimator degrades as the number of RSSs increases.
Fig. 4 compares the ranging transmission energies normalized by the noise energy contained on one sub-carrier in one OFDM symbol duration. The upper subplot shows the average normalized ranging transmission energy per ranging frame for an RSS. The lower subplot shows the average of total normalized ranging transmission energy required for an RSS to finish its ranging process. The proposed method cuts down the ranging energy consumption at an RSS to approximately $\frac{1}{6}$ of that required in the reference method. In addition to the per-frame energy saving, the proposed method requires a smaller number of frames to complete the ranging process which yields further energy saving. For example, with 32 RSSs, the proposed method requires less than $\frac{1}{10}$ of the ranging energy used in the reference method. The close match between simulation and theoretical result in (32) also validates our theoretical analysis of the ranging transmission power.

The theoretical results of the normalized power estimation MSE, calculated by using (24) match very well with the simulation results. The plot is omitted due to the space limitation. The computational complexity for the ranging code detection and timing estimation in one ranging frame for the proposed method is $\frac{1}{2093}$ times the complexity of the reference method. Detailed complexity expressions are skipped due to space limitation. The simulation results demonstrate that the proposed method substantially outperforms the reference method in all considered aspects.

\section{CONCLUSIONS}

We have presented a novel initial ranging method for MIMO-OFDMA systems that takes advantage of multiuser and multi-antenna diversities. By means of ranging signal design, the proposed approach achieves multiuser diversity gain and significant power saving while facilitating efficient low-complexity algorithms for multiuser ranging signal detection, timing offset estimation and power estimation. The proposed ranging method achieves significant gains in ranging signal detection and synchronization performance, tremendous energy saving at the subscriber stations, and huge complexity reduction over the existing ranging methods while utilizing the same amount of time and frequency resources.

\section{REFERENCES}

[1] Broadband Wireless Access: IEEE MAN standard, IEEE LAN/MAN Standards Committee IEEE 802.16a, 2003.

[2] Broadband Wireless Access: IEEE MAN standard, IEEE LAN/MAN Standards Committee IEEE 802.16e, 2005.

[3] J. Krinock, M. Singh, M. Paff, Vincent, A. Lonkar, L. Fung, and C.-C. Lee, "Comments on OFDMA ranging scheme described in IEEE 802.16ab01/01r1," Document IEEE 802.16abc-01/24.

[4] H. Mahmoud, H. Arslan, and M. Ozdemir, "Initial ranging for WiMAX (802.16e) OFDMA," in Proc. IEEE Military Communications Conference, Oct. 2006, pp. 1-7.

[5] X. Fu and H. Minn, "Initial uplink synchronization and power control (ranging process) for OFDMA systems," in Proc. IEEE GLOBECOM, vol. 6, Nov. 29-Dec. 32004 , pp. 3999-4003.

[6] X. Fu, Y. Li, and H. Minn, "A new ranging method for OFDMA systems," IEEE Trans. Wireless Commun., vol. 6, no. 2, pp. 659-669, Feb. 2007. 